\title{
Growth of the thoracic aorta in the smoking population: The Danish Lung Cancer Screening Trial
}

\author{
Lidia R. Bons $^{\mathrm{a}, 1,2}$, Zahra Sedghi Gamechi ${ }^{\mathrm{b}, 1,2}$, Carlijn G.E. Thijssen ${ }^{\mathrm{a}, 1}$, Klaus F. Kofoed ${ }^{\mathrm{c}, 1}$, Jesper H. Pedersen ${ }^{\mathrm{c}, 1}$, \\ Zaigham Saghir $^{\text {d,1 }}$, Johanna J.M. Takkenberg ${ }^{\mathrm{e}, 1}$, Isabella Kardys ${ }^{\mathrm{a}, 1}$, Ricardo P.J. Budde ${ }^{\mathrm{f}, 1}$, \\ Marleen de Bruijne ${ }^{b, g, 1,3}$, Jolien W. Roos-Hesselink ${ }^{\mathrm{a}, *, 1,3}$ \\ a Department of Cardiology, Erasmus Medical Center, Rotterdam, the Netherlands \\ b Biomedical Imaging Group Rotterdam, Departments of Radiology and Medical Informatics, Erasmus Medical Center, Rotterdam, the Netherlands \\ c Department of Cardiothoracic Surgery, Copenhagen University Hospital, Rigshospitalet, Copenhagen, Denmark \\ d Department of Respiratory Medicine, Gentofte University Hospital, Hellerup, Denmark \\ e Department of Cardiothoracic Surgery, Erasmus Medical Center, Rotterdam, the Netherlands \\ ${ }^{\mathrm{f}}$ Department of Radiology and Nuclear Medicine, Erasmus Medical Center, Rotterdam, the Netherlands \\ ${ }^{g}$ Machine Learning Section, Department of Computer Science, University of Copenhagen, Copenhagen, Denmark
}

\section{A R T I C L E I N F O}

\section{Article history:}

Received 19 March 2019

Received in revised form 3 June 2019

Accepted 5 June 2019

Available online $\mathrm{xxxx}$

\section{Keywords:}

Aortic growth

Thoracic aorta

Computed tomography

Smoking

\begin{abstract}
A B S T R A C T
Background: Although the descending aortic diameter is larger in smokers, data about thoracic aortic growth is missing. Our aim is to present the distribution of thoracic aortic growth in smokers and to compare it with literature of the general population.

Methods: Current and ex-smokers aged 50-70 years from the longitudinal Danish Lung Cancer Screening Trial, were included. Mean and 95th percentile of annual aortic growth of the ascending aortic (AA) and descending aortic (DA) diameters were calculated with the first and last non-contrast computed tomography scans during follow-up. Determinants of change in aortic diameter over time were investigated with linear mixed models. Results: A total of 1987 participants (56\% male, mean age $57.4 \pm 4.8$ years) were included. During a median follow-up of 48 months, mean AA and DA growth rates were comparable between males (AA $0.12 \pm 0.31 \mathrm{~mm} /-$ year and DA $0.10 \pm 0.30 \mathrm{~mm} /$ year) and females (AA $0.11 \pm 0.29 \mathrm{~mm} /$ year and DA $0.13 \pm 0.27 \mathrm{~mm} / \mathrm{year}$ ). The 95th percentile ranged from 0.42 to $0.47 \mathrm{~mm} / \mathrm{year}$, depending on sex and location. Aortic growth was comparable between current and ex-smokers and aortic growth was not associated with pack-years. Our findings are consistent with aortic growth rates of 0.08 to $0.17 \mathrm{~mm} /$ years in the general population. Larger aortic growth was associated with lower age, increased height, absence of medication for hypertension or hypercholesterolemia and lower Agatston scores.

Conclusions: This longitudinal study of smokers in the age range of 50-70 years shows that ascending and descending aortic growth is approximately $0.1 \mathrm{~mm} /$ year and is consistent with growth in the general population.
\end{abstract}

(c) 2019 Elsevier B.V. All rights reserved.

\section{Introduction}

'Dilatation of the thoracic aorta is associated with an increased risk of aortic dissection [1], with high mortality rates of up to $50 \%$ in the first 30 days [2]. In addition to the absolute diameter, fast growth of 3$5 \mathrm{~mm} /$ year is mentioned in the guidelines on the diagnosis and treatment of aortic diseases as an important risk factor for dissection and is

\footnotetext{
* Corresponding author at: Cardiology, Erasmus MC, Rg-435, Doctor Molewaterplein 40, 3015 GD Rotterdam, the Netherlands.

E-mail address: j.roos@erasmusmc.nl (J.W. Roos-Hesselink).

1 This author takes responsibility for all aspects of the reliability and freedom from bias of the data presented and their discussed interpretation.

2 L.R.B and Z.S.G shared first authorship.

3 J.W.R. and M.B. shared last authorship.
}

therefore an additional indication to perform preventive surgery $[3,4]$. However, data about risk factors for fast aortic growth is scarce. It has been shown that patients with a bicuspid aortic valve or Marfan syndrome show larger aortic growth rates than the general population [5]. Smoking is associated with larger diameter of the aortic arch and descending aorta [6-8] and with larger aortic growth of the abdominal aorta [9]. Whether smoking is associated with faster thoracic aortic growth is still unknown. With use of a large prospective longitudinal cohort study, the Danish Lung Cancer Screening Trial (DLCST), we aimed to investigate whether aortic growth is larger in current or former smokers when compared to the available cross-sectional studies of the general population. With our longitudinal data of the thoracic aortic growth we will also be able to identify risk factors for fast growth in this subgroup of the population.' 


\section{Methods}

\subsection{Study population}

Participants were recruited from DLCST (www.ClinicalTrials.gov, registration number: NCT 00496977), a randomized controlled trial conducted between 2004 and 2010. Participants in the DLCST volunteered in response to local media advertisements. Current and former smokers aged 50-70 years with at least 20 pack-years and forced expiratory volume in first second (FEV 1 ) of $>30 \%$ of predicted value were included. Participants with body weight above $130 \mathrm{~kg}$, previous treatment for any kind of cancer within 5 years, tuberculosis within 2 years, and any serious illness with life expectancy $<10$ years were excluded. The primary aim of this RCT was to investigate the effect of computed tomography screening on lung cancer mortality. No statistically significant effects of CT screening on lung cancer mortality were found. The study was approved by the National Ethics Committee of Denmark (identification no. H-KA-02045, supplementary protocol 20148) and all participants gave written informed consent. The study design is explained in more detail before [10].

In the DLCST study 2052 participants were randomized to the screening group, which received annual multidetector computed tomography (MDCT) during a 5 year period. This MDCT scans provided the opportunity to perform a post-hoc analysis in which the aortic growth was measured over a long period. For this study we excluded participants with $<1$-year follow-up between the first and last CT scans $(n=65)$, because this follow-up period was too short to accurately measure growth. Overall, 1987 participants were included in the current study.

Clinical characteristics regarding smoking status, history of stroke and ischemic heart disease, medical treatment for diabetes, hypertension or hypercholesterolemia and Agatston calcium scores (of the ascending aorta + arch and of the descending aorta) were collected at baseline as previously defined and described [11]. The Agatston calcium score is a measure of arterial calcium on computed tomography. The calculation is based on the weighted density score given to the highest attenuation value (HU) multiplied by the volume of the calcification. The Agatston score of the ascending aorta, aortic arch and descending aorta were assessed by one observer using Vitrea v. 6.0 (Vital Images, Inc., MN, USA). A standardized procedure for calcium scoring with a threshold of 130 Hounsfield units (HU) was used to identify aortic calcifications.

\subsection{Computed tomography imaging}

All non-ECG-gated, non-contrast CT scans were performed in a single institution with a 16-row Philips Mx8000 MDCT scanner, Philips Medical Systems, Eindhoven, the Netherlands. Scans were performed in supine position after full inspiration in caudocranial scan direction including the entire rib cage and upper abdomen with $120 \mathrm{kV}$ and $40 \mathrm{mAs}$. Scans were performed with spiral data acquisition with the following parameters: section collimation, $16 \times 0.75 \mathrm{~mm}$; pitch, 1.5 ; and rotation time of $0.5 \mathrm{~s}$. The obtained data were reconstructed with a slice thickness of $1 \mathrm{~mm}$ and a hard reconstruction algorithm (Philips D kernel).

\subsection{Measurements of aortic diameter}

Aortic diameters were measured with use of an automatic method, which is validated in 100 participants showing a good agreement with manual aortic diameter measurements [12]. The method combines multi atlas registration to obtain seed points, aorta centerline extraction, and an optimal surface segmentation approach [13] to extract the aorta surface around the centerline. From the extracted 3D aorta segmentation, the average diameters of the ascending aorta and descending aorta at the level of the pulmonary artery bifurcation were computed from the cross-sectional area measured at cross-sectional slices perpendicular to the extracted centerline (Fig. 1). The aortic wall with possible calcification was included in the measurements. In 29 participants, an error occurred in the automatic method for centerline extraction and therefore no aortic diameters were automatically computed. The ascending and descending aortic diameters for these cases were measured manually by drawing the centerline and cross-sectional vessel contour perpendicular to the centerline at the pulmonary bifurcation level as described in detail in our previous work [12]. In the remaining 1958 subjects with accurate centerline extraction we visually checked the following cases to identify inadequate measurements as a results of the automatic method: (1) all outliers of the aortic diameter at baseline and follow-up defined as 2.7 standard deviation above or beneath the median; (2) all subjects who showed aortic growth or decline of $>3.5 \mathrm{~mm}$; and (3) a random sample of 200 images ( 100 baseline and 100 follow-up scans in the same subjects). From the randomly selected 200 scans, only $3(1.5 \%)$ at the ascending aorta and $4(2 \%)$ at the descending aorta showed a slight over or under segmentation. Overall, in 68 subjects adequate measurements of the automatic method were not available due to inadequate segmentations. Also the aortic diameters for these 68 cases were measured manually for both the ascending and descending aorta diameter. As a result, ascending and descending aortic diameters and aortic growth was available in all 1987 participants.

\subsection{Statistical analysis}

Data are expressed as mean \pm SD or as median \pm interquartile range in case the distribution was not normal. Data distribution was checked using histograms. Categorical variables are presented as frequencies with percentages. To present the distribution of annual aortic growth, the annual growth rate was calculated by subtracting the aortic diameter measured on the baseline CT scan from the aortic diameter measured on the last scan during follow-up and subsequently dividing this value by the number of years between the baseline and last follow-up scan. The Student's $t$-test or Mann-Whitney test was used to compare means between two groups at baseline. Comparison of categorical variables was done using the Chi-square test or the Fisher's exact test. For the analyses of Agatston scores, we used natural log-transformed values and added $1.0 \mathrm{~mm}^{3}$ to the nontransformed Agatston values ( $\operatorname{Ln}($ calcification volume +1$)$ ) to deal with values of zero. For pack-years we used the log-transformed values.

To investigate whether change in aortic diameter was associated with baseline characteristics, linear mixed effects (LME) models were used. The ascending and descending aortic diameter were consecutively used as the dependent variable. Time was entered as a random effect. First, all baseline variables were entered concomitantly as independent variables to identify whether they were independently associated with the aortic diameter (while taking into account that the aortic diameter was measured twice in each participant by using the LME). All baseline characteristics (i.e. age, height, weight, sex, medical treatment, medical history, pack years, Agatston scores) were deemed clinically relevant based on previous research $[7,8,14]$. Second, interaction terms of each of the baseline variables with time were entered consecutively into the multivariable model, to assess the independent effect of each of these variables on the change of aortic diameter over time. We also examined the interaction term between time and large aortic diameter (ascending aorta $>40 \mathrm{~mm}$ and descending aorta $>30 \mathrm{~mm}$ ) to assess whether participants with larger aortic diameters show larger changes in aortic diameter over time. All interaction terms, that were found to be significant were presented in the figures. We checked whether the assumptions underlying linear mixed effects modelling (linearity and homoscedasticity) were satisfied.

The IBM SPSS $®$ statistics 21.0 software was used to analyze the data and a p-value of $<0.05$ was considered significant.

\section{Results}

\subsection{Study population}

The baseline characteristics of the 1987 included participants are presented in Table 1 for the total group and separately for males and females. The mean age of our cohort was $57.4 \pm 4.8$ years. Antihypertensive medication was used by $14.8 \%$ of the participants.

\subsection{Aortic diameters and aortic growth}

The distribution of the aortic diameters for both males and females can be found in Supplemental Fig. 1. The ascending and descending aortic diameter at baseline were significantly larger in males (ascending aorta $36.0 \pm 3.5 \mathrm{~mm}$ and descending aorta $28.2 \pm 2.2 \mathrm{~mm}$ ) than in

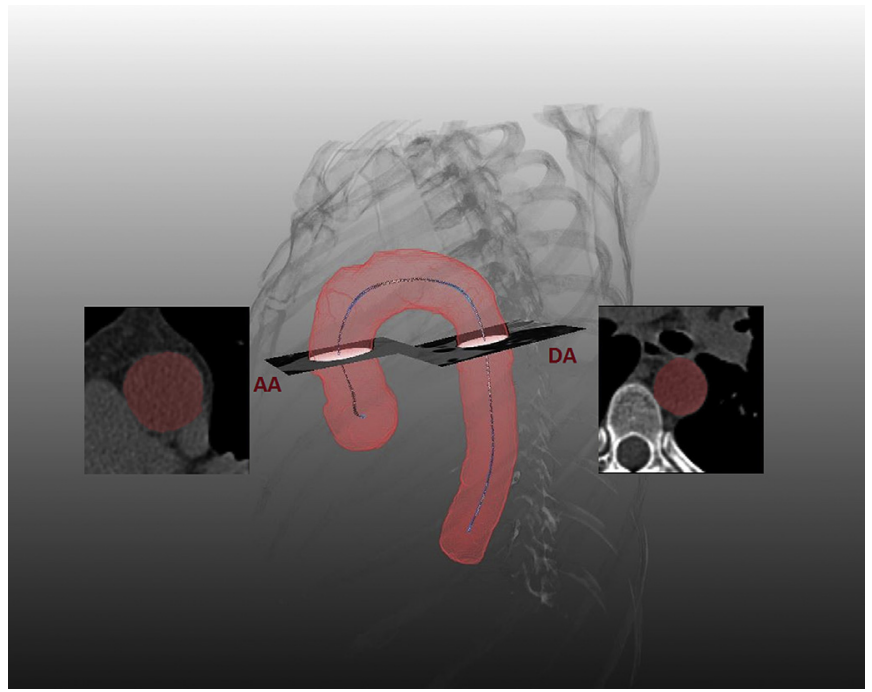

Fig. 1. Measurements of the average ascending and descending aortic diameter. A 3D image of the automatic tool which extracts the centerline (blue) and the surface of the aorta (red) to compute the ascending (AA) and descending aortic (DA) diameters at the level of pulmonary artery bifurcation. Cross-sectional views of the ascending and descending aorta are shown left (AA) and right (DA). Both cross-sections are overlaid with the automatically extracted aortic area (in red). The average diameters is computed as Diameter $=2 \sqrt{\text { Area } / \pi}$. (For interpretation of the references to color in this figure legend, the reader is referred to the web version of this article.) 
females (ascending aorta $33.6 \pm 3.2 \mathrm{~mm}$ and descending aorta $26.1 \pm$ $2.2 \mathrm{~mm}$ ). A baseline aortic diameter of $\geq 40 \mathrm{~mm}$ at the ascending aorta was found in 167 ( $8 \%$ ) participants. For the descending aorta, a baseline aortic diameter of $\geq 40 \mathrm{~mm}$ was found in $1(0 \%)$ participant and $\geq 30 \mathrm{~mm}$ in 257 (13\%) participants. The distribution of annual aortic growth for both males and females is shown in Fig. 2 and was calculated during a median follow-up of 48 months (IQR 47-50 months). Annual growth did not statistically significantly differ between males and females for the ascending aorta (males $0.12 \pm 0.31 \mathrm{~mm} /$ year and females $0.11 \pm$ $0.29 \mathrm{~mm} /$ year) and descending aorta (males $0.10 \pm 0.30 \mathrm{~mm} / \mathrm{year}$ and females $0.13 \pm 0.27 \mathrm{~mm} /$ year). In addition, the aortic growth did not differ significantly between current or former smokers for the ascending aorta (current $0.12 \pm 0.30 \mathrm{~mm} /$ year and former $0.13 \pm$ $0.29 \mathrm{~mm} /$ year) and descending aorta (current $0.11 \pm 0.30 \mathrm{~mm} / \mathrm{year}$ and former $0.11 \pm 0.25 \mathrm{~mm} /$ year). In total, 621 (31\%) participants showed decrease of the ascending aortic diameter in time and 604 (30\%) of the descending aortic diameter. Eighteen people (1\%) had an aortic growth of $>1 \mathrm{~mm} /$ year, which in 9 persons only occurred in the ascending aorta ( 2 former and 7 current smoker), in 6 persons only in the descending aorta (all current smokers) and in 3 persons in both the ascending and descending aorta (one former and two current smokers). In two people $(0.1 \%)>2 \mathrm{~mm} /$ year (both descending aorta) was found and only one $(0.05 \%)$ showed $>3 \mathrm{~mm} /$ year.

\subsection{Determinants of aortic growth}

The association between the baseline characteristics and the aortic diameter is shown in Supplemental Table 1. Higher age, larger height and weight, hypertension and higher Agatston scores were associated with larger ascending aortic diameters, while female and diabetes were associated with smaller ascending aortic diameters. For the descending aorta, higher age, height, weight and Agatston scores were associated with larger aortic diameters, while female and hypercholesterolemia were associated with smaller aortic diameters.

Fig. 3 shows the significance of the interaction terms between baseline variables and time from the linear mixed effects models. Larger height was associated with larger increase in aortic diameter over time. Higher age, hypertension, hypercholesterolemia and Agatston scores were associated with smaller increase in ascending aortic diameter over time. For the descending aorta, higher age, hypertension and higher Agatston score of the descending aorta were associated with smaller change of the descending aorta over time.

\section{Discussion}

This is the first study presenting longitudinal data on sex-specific growth of the ascending and descending aorta in a large population of current or former smokers with at least 20 pack-years. Males showed a growth of $0.12 \pm 0.31 \mathrm{~mm} /$ year for the ascending aorta and $0.10 \pm$ $0.30 \mathrm{~mm} /$ year for the descending aorta. In females, we found a growth of $0.11 \pm 0.29 \mathrm{~mm} /$ year for the ascending aorta and $0.13 \pm 0.27 \mathrm{~mm} /-$ year for the descending aorta. Previous studies showed that smoking is associated with larger diameter of the aortic arch or descending aorta [6-8], suggesting faster growth. As such, it would be expected that the descending aortic growth will also be faster in our study compared to the general population. Nevertheless, our study showed comparable or even smaller growth rates compared to the two largest cross-sectional cohort studies who reported on the association between age and descending aortic diameter. Kalsch et al. [15] calculated an increase of $0.17 \mathrm{~mm}$ for males and 0.16 for females per 1 year increase in age. Wolak et al. [8] showed that the descending aortic diameter was $0.13 \mathrm{~mm}$ larger each 1 year increase in age, which is comparable to our results. Only one study, the Framingham Heart Study [14], measured the thoracic aortic growth longitudinally in a healthy population, but they solely measured the growth at the level of the aortic root. In addition, we have also found no association between pack years and descending aortic growth. Therefore, we can conclude that our data do not support the hypothesis that descending aortic growth would be larger in current or former smokers compared to the general population. Since there is no association found previously between the ascending aortic diameter and smoking, we did not expect any effect of smoking on the ascending aortic growth, which was also confirmed by our results.

The conclusions must be interpreted with caution taking into account the measurement variability of non-ECG-gated non-enhanced $\mathrm{CT}$. In previous literature, the mean intra-observer variation between two measurements of the ascending aorta found in contrast CT scans is found to be $0.1-0.3 \mathrm{~mm}$ for manual measurements [16-18]. Possibly for non-contrast CT scans it is larger. The decrease in AA and DA diameter in $31 \%$ and $30 \%$ of the participants, respectively, is in part caused by this measurement variability. However, the absolute mean difference between the first and last CT scan, not divided by the amount of years in between the two scans, was $0.46 \pm 1.05 \mathrm{~mm}$ for the ascending aorta and $0.44 \pm 0.97 \mathrm{~mm}$ for the descending aorta. This is higher than we would expect based on the intra-observer variability of $0.1-0.3 \mathrm{~mm}$ and therefor our change in aortic diameter could not only be explained by

Table 1

Baseline characteristics.

\begin{tabular}{|c|c|c|c|c|}
\hline & $\begin{array}{l}\text { Total }(\mathrm{n}= \\
1987)\end{array}$ & $\begin{array}{l}\text { Males }(\mathrm{n}= \\
1111)\end{array}$ & $\begin{array}{l}\text { Females ( } \mathrm{n} \\
=876)\end{array}$ & p-Value \\
\hline Age, years & $57.4 \pm 4.8$ & $57.8 \pm 4.8$ & $56.9 \pm 4.8$ & $<0.001$ \\
\hline Height, cm & $173.8 \pm 8.8$ & $179.4 \pm 6.3$ & $166.7 \pm 6.0$ & $<0.001$ \\
\hline Weight, kg & $76.5 \pm 14.2$ & $83.2 \pm 12.1$ & $68.0 \pm 12.0$ & $<0.001$ \\
\hline \multicolumn{5}{|l|}{ Medical treatment } \\
\hline Hypertension, N (\%) & $294(14.8 \%)$ & $158(14.2 \%)$ & $136(15.5 \%)$ & 0.416 \\
\hline Hypercholesterolemia, N (\%) & $168(8.5 \%)$ & $109(9.8 \%)$ & $59(6.7 \%)$ & 0.014 \\
\hline Diabetes, N (\%) & $39(2.0 \%)$ & $30(2.7 \%)$ & $9(1.0 \%)$ & 0.008 \\
\hline History of stroke, N (\%) & $34(1.7 \%)$ & $24(2.2 \%)$ & $10(1.1 \%)$ & 0.082 \\
\hline History of ischemic heart disease, $\mathrm{N}(\%)$ & $40(2.0 \%)$ & $36(3.2 \%)$ & $4(0.5 \%)$ & $<0.001$ \\
\hline Current smoking, $\mathrm{N}(\%)$ & $491(24.7 \%)$ & $274(24.7 \%)$ & $217(24.8 \%)$ & $0.955^{c}$ \\
\hline Pack-years ${ }^{\mathrm{a}}$ & $34(27-42.5)$ & $36(29-46)$ & $31(25.5-39)$ & $<0.001^{\mathrm{b}}$ \\
\hline Agatston score ascending aorta $+\operatorname{arch}^{\mathrm{a}}$ & $36(0-273)$ & $33(0-247)$ & $39.5(0-303.8)$ & $0.795^{\mathrm{b}}$ \\
\hline Agatston score descending aorta ${ }^{a}$ & $0(0-38)$ & $0(0-45)$ & $0(0-25)$ & $0.005^{\mathrm{b}}$ \\
\hline Baseline ascending aortic diameter & $35 \pm 4$ & $36 \pm 3$ & $34 \pm 3$ & $<0.001$ \\
\hline Baseline descending aortic diameter & $27 \pm 2$ & $28 \pm 2$ & $26 \pm 2$ & $<0.001$ \\
\hline
\end{tabular}

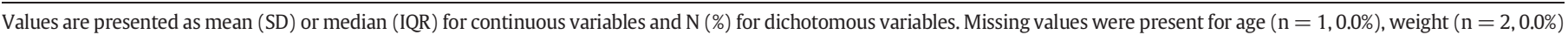
and Agatston scores of the aorta $(n=8,0.4 \%)$.

a Nontransformed median score with interquartile range.

b Mann-Whitney test.

c Fisher's exact test. 

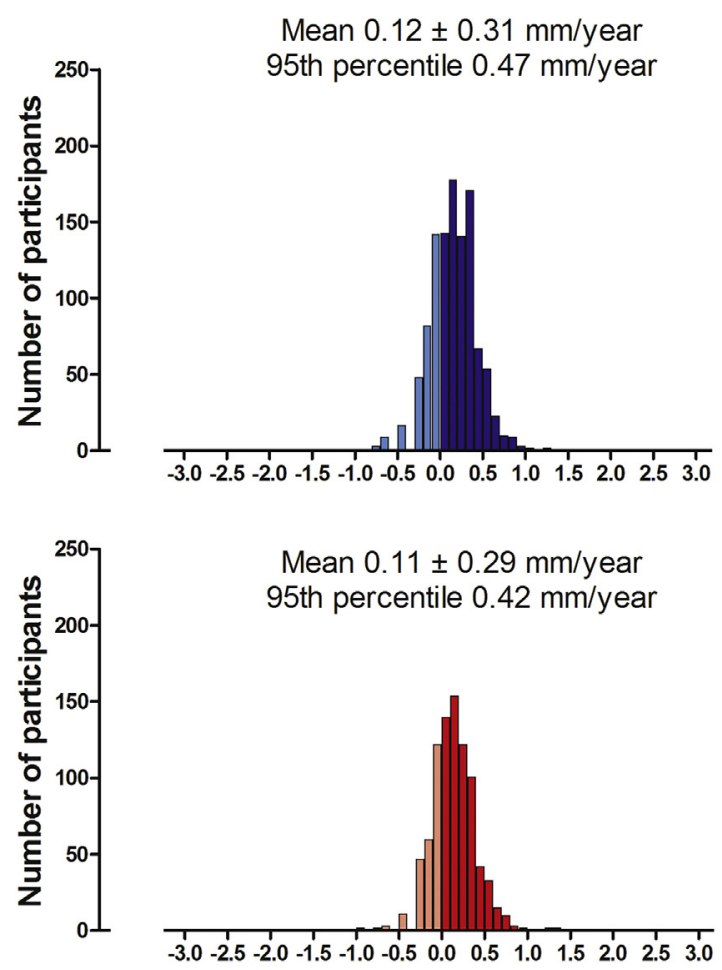

Ascending aorta
Mean $0.10 \pm 0.30 \mathrm{~mm} /$ year

95th percentile $0.43 \mathrm{~mm} /$ year

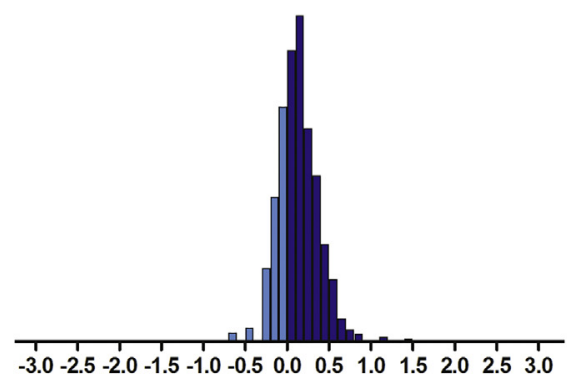

Mean $0.13 \pm 0.27 \mathrm{~mm} /$ year

95th percentile $0.42 \mathrm{~mm} /$ year

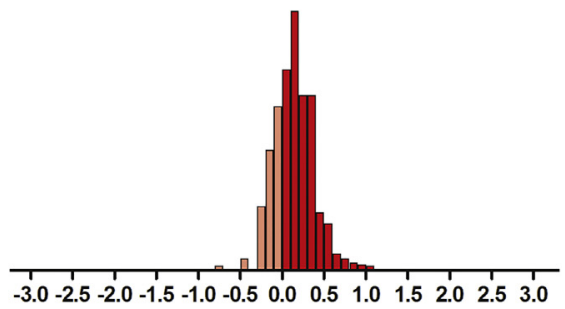

Descending aorta

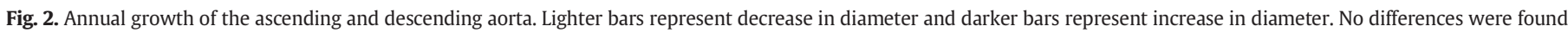
between males and females in ascending aortic growth $(\mathrm{p}=0.394)$ and descending aortic growth $(\mathrm{p}=0.087)$.

measurement variability. Moreover, the use of identical CT scanners and automated segmentation for both baseline and follow-up measurements is an important strength of this study because it prevented us from additional inter-observer and inter-modality variability.

\subsection{Determinants of aortic growth}

From previous literature we know that body measurements are important in the assessment of aortic diameters $[19,20]$. For instance, in Turner patients with typically a short stature, the use of the aortic size index (ASI) is advised, which corrects for body surface area [21]. For the aortic growth, little data is available on the effect of body measurements. The Framingham Heart Study [14] included a slightly younger population (mean age $50 \pm 14$ years) with comparable BMI (25.5 \pm $4.4 \mathrm{~kg} / \mathrm{m}^{2}$ ) and showed that BMI was correlated with change in aortic root diameter over time. We examined height and weight separately and showed that the effect of body measures on the ascending aortic growth is mainly based on height.

Higher age was associated with both less ascending and descending aortic growth. Aortic remodeling over the adult life is accompanied by reduced aortic elasticity [22] and reduced tortuosity with increased curvature [23]. Because of these changes, one may expect aortic growth will decrease at older age and aortic diameters will stabilize. Treatment for hypertension was associated with slower ascending and descending aortic growth. Since higher blood pressure is associated with larger descending aortic diameters [7], we would assume that participants with hypertension would show larger descending aortic growth. However, patients being treated for hypertension may represent the group with controlled blood pressure and the group of patients who are not receiving treatment may contain patients with uncontrolled blood pressure. Because we had no information about the exact blood pressure, which is a limitation of this study, we could not verify this assumption. This could also be the case with hypercholesterolemia because patients with treatment for hypercholesterolemia showed a smaller increase in aortic diameter. An ascending aortic diameter of $>40 \mathrm{~mm}$ or descending aortic diameter of $>30 \mathrm{~mm}$ was not associated with change in diameter over time in our cohort. Although patients with aortic aneurysms show larger growth rates [24], we may have had too few patients with aortic dilatation in our cohort to prove this.

A recent systematic review, which included all causes of thoracic aortic aneurysms, showed a mean growth rate in patients from 0.2 to $2.8 \mathrm{~mm} /$ year for ascending aorta and aortic arch, while those for descending and aorta ranged from 1.9 to $3.4 \mathrm{~mm} /$ year [24]. Detaint et al. [5] observed at the level of the ascending aorta an aortic growth of $0.12 \pm 1.0 \mathrm{~mm} /$ year in Marfan syndrome and $0.42 \pm 0.6 \mathrm{~mm} /$ year in BAV. These growth rates of patients with a bicuspid aortic valve or degenerative aortapathy are larger than found in our cohort with current and ex-smokers. In current guidelines for thoracic aortic diseases, different definitions are used for extensive growth ( $\geq 3 \mathrm{~mm}$ or $\geq 5 \mathrm{~mm}$ ), which warrants preventive surgery. Our study showed only two cases with growth $>2 \mathrm{~mm} /$ year and only one with $>3 \mathrm{~mm} / \mathrm{year}$, which suggests that extensive growth, defined by the guidelines, is relatively rare in the general smoking population. Based on our 95th percentiles, annual aortic growth of $0.5 \mathrm{~mm}$ is the upper limit of normal in current or former smokers.

\subsection{Limitations}

One large limitation of our study is that we did not include our own reference group of healthy subjects. The literature only contained cross-sectional data with the mean thoracic aortic growth rate of the general population and therefore we could not compare the distribution (95th percentile) of aortic growth rates in our group with a reference group. Another limitation is the lack of information about diseases related to aortic pathology, such as connective tissue disease and bicuspid aortic valve. This 
$p$-value for interaction term

\section{Subgroup}

Aged $<=57$ year
Aged $>57$ years

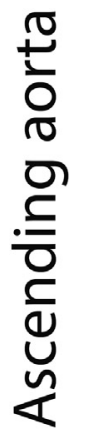

Height $<=174 \mathrm{~cm}$

Height $>174 \mathrm{~cm}$

No hypertension

Hypertension

No hypercholesterolemia Hypercholesterolemia

Agatston score ascending aorta + arch

Score 0

Score more than 0

$0.122(0.110-0.134)$

$0.076(0.032-0.121)$

Agatston score descending aorta

Score 0

Score more than 0

$0.122(0.110-0.134)$

$0.076(0.032-0.121)$

$0.113(0.098-0.129)$

$0.123(0.106-0.140)$

$0.124(0.111-0.137)$

$0.085(0.054-0.115)$

$0.122(0.110-0.134)$

$0.076(0.032-0.121)$ change in diameter over time

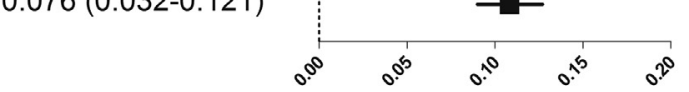

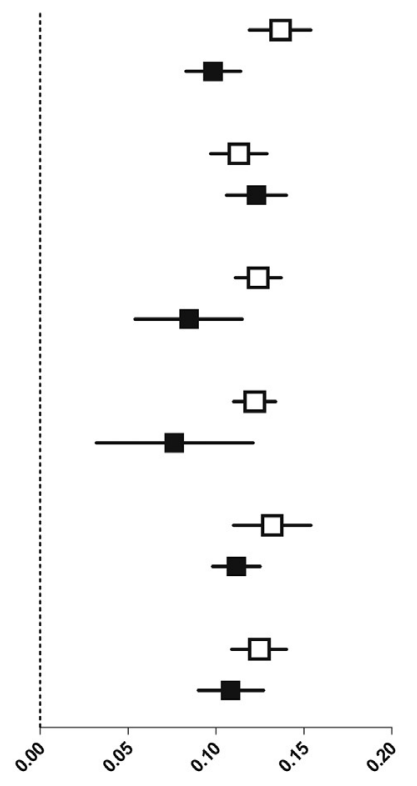

$0.001^{*}$

0.007

0.019

0.034

0.006

0.022

0.011 $0.098(0.083-0.114)$

Aged $>57$ years

$0.124(0.111-0.137)$

$0.085(0.054-0.115)$

No hypertension

0.085

Agatston score descending aorta

Score 0

$0.122(0.110-0.134)$

Score more than 0

$0.076(0.032-0.121)$
$<0.001$

$<0.001$

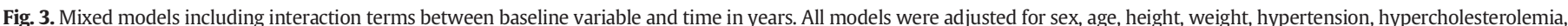

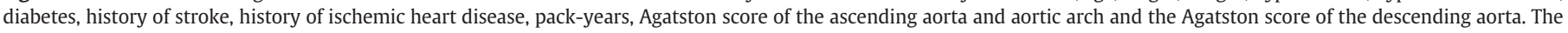

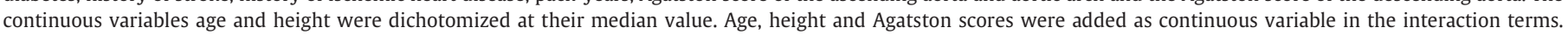
*Interpretation: A higher age is associated with less increase in the ascending aortic diameter over time (in years).

information was not available because the primary aim of this RCT was to investigate the effect of computed tomography screening on lung cancer mortality. Because this study was a post-hoc analysis, thoracic aortic growth was neither a primary nor a secondary outcome measure of the original trial. The limited age range of 50-70 years also prevents the generalization of our results to the total population. In addition, the limited aortic growth may have limited the power of our analysis. However, our cohort was large enough to find a significant aortic growth for both the ascending and descending aorta and also several determinants were found to be significant associated with the change in thoracic aortic diameter over time. Another limitation of this study is the use of non-ECG gated, non-contrast CT scans. Non-gated CT scans show significant more motion artifacts than ECG-gated CT scans [25], which likely effect aortic measurements. The use of contrast-enhanced CT is preferred for thoracic aortic measurements but could cause unnecessary complications. However, both baseline and follow-up measurements were made in the same manner.

An issue that warrants consideration in our study is the fact that we examined a total of 13 variables. If we were to account for multiple testing using a Bonferroni correction, only age would remain statistically significant for the ascending aorta, while hypertension and Agatston score would remain statistically significant for the descending aorta. However, our study was not data driven but hypothesis driven; the choice of variables we investigated was based on previous findings from the literature. These variables were thus already implicated in the disease process by earlier studies. Correcting for multiple testing in spite of this hypothesis driven approach could result in failure to recognize potentially interesting factors. In any case, our findings may be considered as indicative of a potential association, and these hypothesis generating findings merit validation in other large studies.

\section{Conclusion}

This longitudinal study of current and ex-smokers shows that the ascending and descending aorta grows on average $0.1 \mathrm{~mm} /$ year in both males and females in the age range of 50-70 years. The aortic growth rates are consistent (or even smaller) with the numbers available in cross-sectional studies of the general population. According to the 95th percentile, an aortic growth of $>0.5 \mathrm{~mm} /$ year can be considered 
the upper limit of normal. Larger change of aortic diameters in time was associated with lower age, increased height, absence of medication for hypertension or hypercholesterolemia, lower Agatston score and a large thoracic aortic diameter.

Supplementary data to this article can be found online at https://doi. org/10.1016/j.ijcard.2019.06.010.

\section{Funding}

This study was supported by grants from the Dutch Heart Foundation (2003B179) and ZonMw (84920001). In addition, this study was funded by the Iranian Ministry of Science Research and Technology (MSRT), the Netherlands Organisation for Scientific Research (NWO). The Danish Lung Cancer Screening Trial (DLCST) was funded by the Danish Ministry of Interior and Health. The funding organizations had no role in the design and conduct of the study; collection, management, analysis, and interpretation of the data; preparation, review, or approval of the manuscript; or decision to submit the manuscript for publication.

\section{Declaration of Competing Interest}

All other authors have reported that they have no relationships relevant to the contents of this paper to disclose.

\section{Acknowledgements}

The authors thank the investigators, staff, and participants of the Danish Lung Cancer Screening Trial for their valuable contributions.

\section{References}

[1] R.R. Davies, L.J. Goldstein, M.A. Coady, S.L. Tittle, J.A. Rizzo, G.S. Kopf, et al., Yearly rupture or dissection rates for thoracic aortic aneurysms: simple prediction based on size, Ann. Thorac. Surg. 73 (2002) 17-27 (discussion -8).

[2] I.H. Melvinsdottir, S.H. Lund, B.A. Agnarsson, K. Sigvaldason, T. Gudbjartsson, A. Geirsson, The incidence and mortality of acute thoracic aortic dissection: results from a whole nation study, Eur. J. Cardiothorac. Surg. 50 (2016) 1111-1117.

[3] R. Erbel, V. Aboyans, C. Boileau, E. Bossone, R.D. Bartolomeo, H. Eggebrecht, et al., 2014 ESC Guidelines on the diagnosis and treatment of aortic diseases: Document covering acute and chronic aortic diseases of the thoracic and abdominal aorta of the adult. The Task Force for the Diagnosis and Treatment of Aortic Diseases of the European Society of Cardiology (ESC), Eur. Heart J. 35 (2014) 2873-2926.

[4] S.A. Goldstein, A. Evangelista, S. Abbara, A. Arai, F.M. Asch, L.P. Badano, et al. Multimodality imaging of diseases of the thoracic aorta in adults: from the American Society of Echocardiography and the European Association of Cardiovascular Imaging: endorsed by the Society of Cardiovascular Computed Tomography and Society for Cardiovascular Magnetic Resonance, J. Am. Soc. Echocardiogr. 28 (2015) 119-182.

[5] D. Detaint, H.I. Michelena, V.T. Nkomo, A. Vahanian, G. Jondeau, M.E. Sarano, Aortic dilatation patterns and rates in adults with bicuspid aortic valves: a comparative study with Marfan syndrome and degenerative aortopathy, Heart 100 (2014) 126-134.

[6] Y. Agmon, B.K. Khandheria, I. Meissner, G.L. Schwartz, J.D. Sicks, A.J. Fought, et al., Is aortic dilatation an atherosclerosis-related process? Clinical, laboratory, and transesophageal echocardiographic correlates of thoracic aortic dimensions in the population with implications for thoracic aortic aneurysm formation, J. Am. Coll. Cardiol. 42 (2003) 1076-1083.
[7] B. Mensel, L. Hesselbarth, M. Wenzel, J.P. Kuhn, M. Dorr, H. Volzke, et al., Thoracic and abdominal aortic diameters in a general population: MRI-based reference values and association with age and cardiovascular risk factors, Eur. Radiol. 26 (2016) 969-978.

[8] A. Wolak, H. Gransar, L.E. Thomson, J.D. Friedman, R. Hachamovitch, A. Gutstein, et al., Aortic size assessment by noncontrast cardiac computed tomography: normal limits by age, gender, and body surface area, JACC Cardiovasc. Imaging 1 (2008) 200-209.

[9] A.R. Brady, S.G. Thompson, F.G. Fowkes, R.M. Greenhalgh, J.T. Powell, U.K.S.A.T Participants, Abdominal aortic aneurysm expansion: risk factors and time intervals for surveillance, Circulation 110 (2004) 16-21.

[10] J.H. Pedersen, H. Ashraf, A. Dirksen, K. Bach, H. Hansen, P. Toennesen, et al., The Danish randomized lung cancer CT screening trial-overall design and results of the prevalence round, J. Thorac. Oncol. 4 (2009) 608-614.

[11] T. Rasmussen, L. Kober, J.H. Pedersen, A. Dirksen, L.H. Thomsen, S. Stender, et al., Relationship between chronic obstructive pulmonary disease and subclinical coronary artery disease in long-term smokers, Eur. Heart J. Cardiovasc. Imaging 14 (2013) 1159-1166.

[12] Z. Sedghi Gamechi, L.R. Bons, M. Giordano, D. Bos, R.P.J. Budde, K.F. Kofoed, et al., Automated 3D segmentation and diameter measurement of the thoracic aorta on noncontrast enhanced CT, Eur. Radiol. (2019).

[13] J. Petersen, M. Nielsen, P. Lo, L.H. Nordenmark, J.H. Pedersen, M.M. Wille, et al., Optimal surface segmentation using flow lines to quantify airway abnormalities in chronic obstructive pulmonary disease, Med. Image Anal. 18 (2014) 531-541.

[14] C.S. Lam, V. Xanthakis, L.M. Sullivan, W. Lieb, J. Aragam, M.M. Redfield, et al., Aortic root remodeling over the adult life course: longitudinal data from the Framingham Heart Study, Circulation 122 (2010) 884-890.

[15] H. Kalsch, N. Lehmann, S. Mohlenkamp, A. Becker, S. Moebus, A. Schmermund, et al., Body-surface adjusted aortic reference diameters for improved identification of patients with thoracic aortic aneurysms: results from the population-based Heinz Nixdorf Recall study, Int. J. Cardiol. 163 (2013) 72-78.

[16] J.F. Rodriguez-Palomares, G. Teixido-Tura, V. Galuppo, H. Cuellar, A. Laynez, L. Gutierrez, et al., Multimodality Assessment of Ascending Aortic Diameters: Comparison of Different Measurement Methods, J. Am. Soc. Echocardiogr. 29 (2016) 819-826 (e4).

[17] L.E. Quint, P.S. Liu, A.M. Booher, K. Watcharotone, J.D. Myles, Proximal thoracic aortic diameter measurements at CT: repeatability and reproducibility according to measurement method, Int. J. Card. Imaging 29 (2013) 479-488.

[18] L.R. Bons, A.L. Duijnhouwer, S. Boccalini, A.T. van den Hoven, M.J. van der Vlugt, R.G. Chelu, et al., Intermodality variation of aortic dimensions: How, where and when to measure the ascending aorta, Int. J. Cardiol. (2018).

[19] R.R. Davies, A. Gallo, M.A. Coady, G. Tellides, D.M. Botta, B. Burke, et al., Novel measurement of relative aortic size predicts rupture of thoracic aortic aneurysms, Ann. Thorac. Surg. 81 (2006) 169-177.

[20] M.A. Zafar, Y. Li, J.A. Rizzo, P. Charilaou, A. Saeyeldin, C.A. Velasquez, et al., Height alone, rather than body surface area, suffices for risk estimation in ascending aortic aneurysm, J. Thorac. Cardiovasc. Surg. 155 (2018) 1938-1950.

[21] C.H. Gravholt, N.H. Andersen, G.S. Conway, O.M. Dekkers, M.E. Geffner, K.O. Klein, et al., Clinical practice guidelines for the care of girls and women with Turner syndrome: proceedings from the 2016 Cincinnati International Turner Syndrome Meeting, Eur. J. Endocrinol. 177 (2017) G1-G70.

[22] A. Redheuil, W.C. Yu, C.O. Wu, E. Mousseaux, A. de Cesare, R. Yan, et al., Reduced ascending aortic strain and distensibility: earliest manifestations of vascular aging in humans, Hypertension 55 (2010) 319-326.

[23] D. Craiem, G. Chironi, A. Redheuil, M. Casciaro, E. Mousseaux, A. Simon, et al., Aging impact on thoracic aorta 3D morphometry in intermediate-risk subjects: looking beyond coronary arteries with non-contrast cardiac CT, Ann. Biomed. Eng. 40 (2012) 1028-1038

[24] D. Oladokun, B.O. Patterson, J. Sobocinski, A. Karthikesalingam, I. Loftus, M.M Thompson, et al., Systematic Review of the Growth Rates and Influencing Factors in Thoracic Aortic Aneurysms, Eur. J. Vasc. Endovasc. Surg. 51 (2016) 674-681.

[25] J.E. Roos, J.K. Willmann, D. Weishaupt, M. Lachat, B. Marincek, P.R. Hilfiker, Thoracic aorta: motion artifact reduction with retrospective and prospective electrocardiography-assisted multi-detector row CT, Radiology 222 (2002) 271-277. 